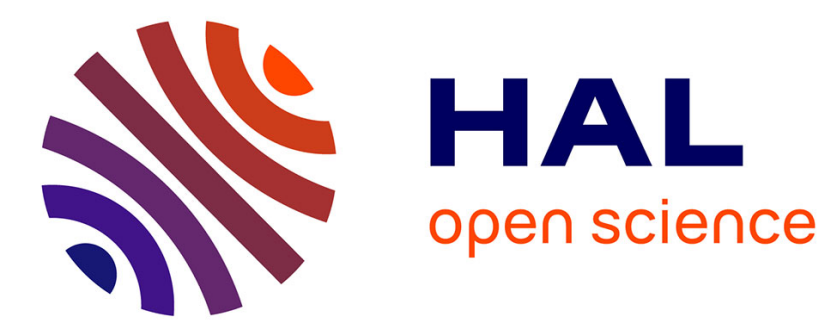

\title{
A two-color planar LIF technique to map the temperature of droplets impinging onto a heated wall
}

Pierre Dunand, Guillaume Castanet, Fabrice Lemoine

\section{To cite this version:}

Pierre Dunand, Guillaume Castanet, Fabrice Lemoine. A two-color planar LIF technique to map the temperature of droplets impinging onto a heated wall. Experiments in Fluids, 2012, 52 (4), pp.843856. 10.1007/s00348-011-1131-1 . hal-01499163

\section{HAL Id: hal-01499163 https://hal.science/hal-01499163}

Submitted on 31 Mar 2017

HAL is a multi-disciplinary open access archive for the deposit and dissemination of scientific research documents, whether they are published or not. The documents may come from teaching and research institutions in France or abroad, or from public or private research centers.
L'archive ouverte pluridisciplinaire HAL, est destinée au dépôt et à la diffusion de documents scientifiques de niveau recherche, publiés ou non, émanant des établissements d'enseignement et de recherche français ou étrangers, des laboratoires publics ou privés.

\section{(ㅇ)(1) $\$$}

Distributed under a Creative Commons Attribution - NonCommercial - NoDerivatives $\mid 4.0$ 


\title{
A two-color planar LIF technique to map the temperature of droplets impinging onto a heated wall
}

\author{
P. Dunand - G. Castanet $\cdot$ F. Lemoine
}

\begin{abstract}
This work consists in the development of the planar extension of two-color LIF, usually working as a point-wise technique. This latter has already demonstrated its ability to characterize the temperature of droplets in various situations including droplet evaporation in combusting flows or droplet/wall interactions in the case of point-wise measurements. This technique is based on the measurement of the relative intensity detected on two adequate spectral bands of a single fluorescent tracer. It allows absolute temperature measurement when a reference at a given temperature is known. The two-color LIF system is designed for observing single drop impacts onto a hot wall with a field of view limited to a few square millimeter. In this study, the focus is placed first on the description of the technique development: the selection of a suitable tracer, its temperature calibration, the correction for the non-linearity of the response of the measurement system, and the pixel-by-pixel correspondence of the camera images. After several tests carried out on droplets in temperature-controlled conditions, the feasibility of the method is finally demonstrated in the case of droplets impinging on a heated wall for different impact regimes: rebound, splashing, and deposition of a boiling liquid film.
\end{abstract}

\section{Introduction}

Liquid cooling is widely used in application areas where the required dissipative power is very large. The cooling

P. Dunand · G. Castanet $(\bowtie) \cdot$ F. Lemoine

LEMTA, Nancy-Université, CNRS, 2 avenue de la Forêt de

Haye BP 160, 54504 Vandœuvre-lès-Nancy Cedex, France

e-mail: guillaume.castanet@ensem.inpl-nancy.fr efficiency is particularly high due to vaporization. While pool boiling and jet impingement techniques have satisfactorily provided high heat dissipation rates, they have generally failed to insure uniform and controlled cooling because of large spatial variations in surface extracted heat flux. Not only the quality of this cooling can be a problem but also its consumption of massive quantities of water and energy. These features are much less of a problem when dealing with sprays. However, the industrial integration of spray cooling remains fairly limited when large power dissipations are required as in steel processing. This is mainly due to poor understanding of the complex flow fields and heat transfer characteristics of spray/wall interactions. In most experimental studies, heat transfers were characterized on the solid side, usually using embedded thermocouples. However, these measurements gave limited insights into the phenomena occurring within the liquid phase.

In the present study, the emphasis is placed into the measurement of the liquid variation in temperature when droplets interact with a hot solid surface. Measuring the temperature of droplets is not a novelty. Although several difficulties remain, different optical techniques have been developed in the past decades to characterize the droplet temperature in sprays or in individual droplet streams. The rainbow refractometry consists in observing the angular position of the monochromatic rainbow which depends on the refractive index and hence the liquid temperature (Van Beeck and Riethmuller 1995; Walker 1976). However, a perfect sphericity of the droplet is required to obtain a correct temperature after inversion. This limits the capabilities of this method to handle conditions where the droplet undergoes deformations like in droplet/wall interactions. The transient cooling of an evaporating water droplet, suspended in a dry-air jet, was also determined 
using thermochromic liquid crystal thermography (Richards and Richards 1998). Other methods were based on the laser-induced fluorescence (LIF) of dye tracers. Laserinduced exciplex fluorescence (LIEF) was used to characterize the transient temperature field within falling droplets (Lu and Melton 2000). The ratio of exciplex and monomer in the liquid phase depends on the local temperature. However, an oxygen-free environment is required due to the strong quenching of the exciplex by oxygen. The twocolor laser-induced fluorescence (2cLIF) was developed specifically to measure the temperature of single-component droplets in either inert or reactive flows (Lavieille et al. 2001). It was also extended to handle the case of multi-component droplets (Deprédurand et al. 2008; Maqua et al. 2006). This method is a two-color one-singledye approach, i.e., the emissions from two different spectral bands with different temperature sensitivities are used. The ratio of the fluorescence intensity of these two bands allows eliminating the effects of parameters that are unknown or difficult to control such as the variations in laser intensity, tracer concentration, or measurement volume during the acquisitions. Being in principle insensitive to the shape of the liquid volume, the 2cLIF technique is particularly well adapted to handle complex situations with droplet deformations.

Recently, this technique was used to determine the droplet change in temperature during their impingement onto a heated solid surface (Castanet et al. 2009). A linear monodisperse stream of water droplets was impacted onto a nickel plate, the temperature of which can be set above the Leidenfrost point. A series of point-wise measurements were performed near the location of the droplet impact in order to determine the average heating of the droplets during their transit close to the wall. Results reveal a clear correlation between the droplet heating and the incident Weber number based on the normal component of the droplet velocity. This application of the $2 \mathrm{cLIF}$ thermometry was restricted to pointwise measurements which imply a cumbersome point-bypoint scanning to reconstruct the temperature distribution in the liquid phase of the flow. This paper presents an extension of the 2 cLIF technique to planar laser-induced fluorescence (PLIF) in order to obtain the temperature field of the droplets impinging onto a heated wall.

PLIF techniques have been used in liquid flows to characterize temperature fields. Coolen et al. (1999) visualized the temperature in a natural convection flow by means of rhodamine B. In this experiment, almost the full emission spectrum of rhodamine $B$ is detected by a single camera. In such a single-color/single-dye technique, one of the main difficulties is that any spatial and temporal variations in the local incident laser intensity need to be separately accounted for, a condition that is almost impossible to satisfy if the variations are caused by the flow itself. For example, in the case of droplets, the local incident laser intensity is modified by the shapes, the sizes, and the positions of the droplets within the laser sheet. For quantitative measurements, another requirement is that the tracer concentration remains constant. This is not possible when droplets evaporate, since the organic dyes used in the liquids (rhodamine, fluorescein,...) are very weakly volatile.

A common method to eliminate these problems is to use a ratiometric method. One possibility is the two-color two-dye approach where the flow is seeded with two dyes (Coppeta and Rogers 1998; Kim et al. 2003; Sakakibara and Adrian 1999, 2004; Shafii et al. 2010). Coppeta and Rogers (1998) make a comprehensive description of the technique and demonstrate its feasibility, not only for temperature but also for $\mathrm{pH}$ determination. Sakakibara and Adrian $(1999,2004)$ applied the technique to perform measurements in thermal convection fields. Kim et al. (2003) have extended the method at the microscopic scale. While most of the measurements at larger scales were carried out using two cameras and a dichroic beam splitter at $45^{\circ}$ incident angle, they used a single-camera system and the detection of the fluorescence was done sequentially by alternating two band-pass filters. This avoids the problem of the pixel-by-pixel correspondence of the images of the cameras. To achieve accuracy in the two-color two-dye approach, one of the dyes should be sensitive to temperature and the other should not or only very weakly while the emission spectra of the two dyes should differ enough to permit the separation of the emitted light by optical means. Among the difficulties related to this approach, the concentration ratio of the dyes must be kept constant during the experiments. Moreover, the emission of one dye can be partially absorbed by the second dye. This phenomenon is generally referred as a spectral conflict. As a result, the detected emission intensity is dependent on the optical path length within the liquid.

Another possible approach is the already-mentioned twocolor/single-dye strategy (Lavieille et al. 2001). In its PLIF form, the implementations of this method were relatively limited. A demonstration was made in a single-phase liquid flow (Bruchhausen et al. 2005). In regard to droplets and sprays, measurements have been reported by Düwel et al. (2007) in the case of burning ethanol droplets seeded with rhodamine B. In this work, two intensified CCD cameras are positioned on both sides of a spray burner. Because of the large field of view, about $4 \mathrm{~cm}$, the pixel-by-pixel correspondence between the two cameras does not seem to be a problem. In the following, the emphasis will be placed on the development of a two-color/single-dye PLIF system designed to measure the temperature distribution within a 
stream of droplets at a small spatial scale limited to a few millimeters.

\section{Droplet generation and time-resolved shadowgraphy}

In order to study droplet/wall interactions, an experimental setup was specifically designed. Regarding the heated plate and the droplet injector, the experimental setup is very similar to that used in a previous study (Castanet et al. 2009). A sketch of the experimental setup is presented in Fig. 1. The heated plate is a square parallelepiped $\left(2 \times 2 \times 10 \mathrm{~cm}^{3}\right)$ made of nickel. An electrical resistance $(110 \Omega)$ is inserted in its center, and the plate is heated by the energy dissipated into the resistance. A power regulator allows adjusting the voltage supplied to the resistance in order to reach a set point temperature in the core of the plate where a thermocouple is positioned. Due to the high thermal conductivity of nickel $\left(\lambda=50 \mathrm{~W} \mathrm{~m}^{-1} \mathrm{~K}^{-1}\right)$, the temperature at the surface of the heated sample is almost homogeneous (Castanet et al. 2009). The wall temperature can be set to a value much higher than the Leidenfrost temperature for which a vapor film forms almost instantaneously between the liquid water and the wall. Presently, this occurs around $220^{\circ} \mathrm{C}$ for a water sessile droplet.

Additionally, a linear monodisperse droplet stream is created by the disintegration of a cylindrical liquid jet. The breakup is driven by a Rayleigh-type instability that can be triggered by mechanical vibrations from a piezoceramic. For some specific frequencies of the vibrations, the liquid jet splits into equally spaced and monosized droplets. The size of the injector orifice and the inlet pressure can be changed from an experiment to another which allows to some extents adjusting separately the size $D$, the frequency $f$, and the velocity $V$ of the droplets. The droplet generator can be rotated precisely to any prescribed angle $\alpha$ of incidence.

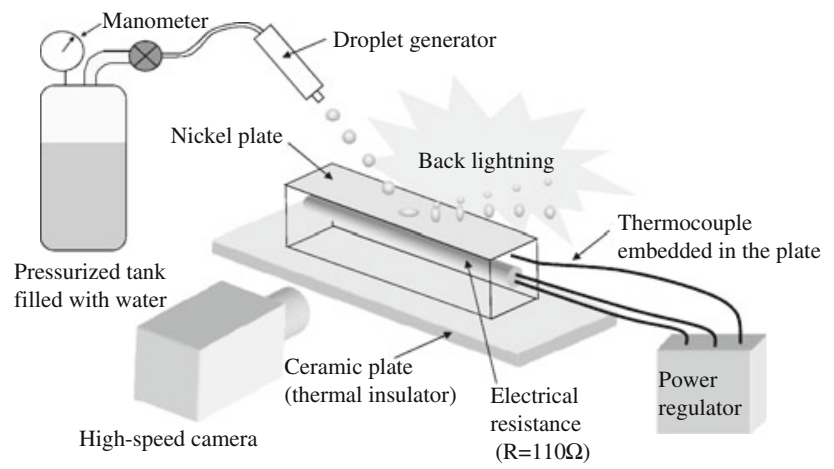

Fig. 1 Schematic view of the experimental setup and the optical layout for shadow imagery
The temperature of the injector body $T_{\mathrm{inj}}$ is regulated while the liquid temperature is measured by a thermocouple placed just before the orifice of the injector. Knowing the injection frequency, the initial droplet size can be determined very accurately from the measurement of the liquid flowrate.

A high-speed (HS) camera is used to visualize the impact of individual droplet onto the heated wall. The HS camera is a Phantom v710 equipped with a 12-bits CMOS sensor that can provide up to $7,500 \mathrm{fps}$ at full resolution $(1,280 \times 800$ pixels $)$. It is used with a reduced resolution $(512 \times 128$ pixels $)$ which enables to perform the image acquisition at a much higher frame rate, typically in the order of 100,000 fps. This is sufficient to resolve in time the droplet/wall interactions in the experimental conditions encountered in this study. For example, a $150-\mu \mathrm{m}$-diameter droplet in the bouncing regime has a resident time (duration of the contact between the droplet and the wall) of about $150 \mu \mathrm{s}$, meaning that the impact process is segmented into about 15 frames. As presented in Fig. 1, the droplets are illuminated from behind using a very bright light source (a 400-W HMI lamp with a parabolic reflector). Sharply contrasted images of the droplets are obtained by shadow imaging while the shuttering of the camera is maintained around $1 \mu$ s to avoid any motion blur. A zoom lens allows observing in detail the droplet deformation and possible splashing, with a field of view ranging from $400 \mu \mathrm{m}$ to $2 \mathrm{~mm}$. The images are then processed with a home-made tracking program in order to determine the main features of the impact such as the incident angle, the normal and tangential velocities, the residence time, or the spreading diameter of the droplets.

\section{The two-color PLIF technique}

\subsection{Principles of the two-color laser-induced fluorescence}

The 2 cLIF thermometry is based on the measurement of the fluorescence intensity of a single-dye tracer. In liquids, the fluorescence quantum yield $\eta$ is strongly influenced by the quenching, which depends on the temperature, while the absorption cross-section of the dye molecule may also exhibit a significant sensitivity to the temperature like fluorescein 27 (Sutton et al. 2008). This section gives an overview of the principles of this method. For more details, the reader can refer to previous papers (Lavieille et al. 2001; Lemoine et al. 1999).

A laser beam induced the fluorescence of a dye dissolved into a liquid medium. The fluorescence signal $I$ emerging from the medium toward the detector can be expressed as: 


$$
I(\lambda)=K_{\text {opt }}(\lambda) \varepsilon_{0} I_{0} c V \eta \exp \left(-c\left(\varepsilon_{0} a+\varepsilon(\lambda) b\right)\right),
$$

where $K_{\text {opt }}$ is a parameter depending on the optical properties of the detection system (e.g., the solid angle of the detection and the spectral sensitivity of the detectors). $c$ is the concentration in dye molecules and the product $c . V$ corresponds to the number of molecules that are illuminated by the laser beam while in the field of view of the detector. $I_{0}$ is the intensity of the laser beam before crossing the absorbing medium. The laser intensity in the absorbing medium is affected by the absorption of the incident laser beam, and the fluorescence emission can be partially reabsorbed. $a$ and $b$ denote the optical path length of the laser beam and the fluorescence in the liquid medium. $\varepsilon_{0}$ is the coefficient of absorption at the wavelength of the laser and $\varepsilon(\lambda)$ at a given wavelength of the fluorescence spectrum. If absorption can be neglected, Eq. 1 simplifies into:

$I(\lambda)=K_{\text {opt }}(\lambda) \varepsilon_{0} I_{0} c V \eta$.

The temperature dependence of the fluorescent emission appears on the one hand in the absorption coefficient $\varepsilon_{0}$ and on the other hand in the fluorescence quantum yield $\eta$. The fluorescence quantum yield is defined by $\eta=A / A+Q$ where $A$ is the Einstein coefficient for spontaneous emission and $Q$ the electronic quenching rate. The quenching rate is proportional to the concentration in quenchers $[M]$, which is assumed to be constant. According to Glowacki (1964), the quenching rate can be given by:

$Q=k q_{0}[M] \exp \left(-E_{a} / k T\right)$.

where $E_{a}$ is the activation energy involved in collisions between a fluorescent molecule and a molecule of the solvent and $k q_{0}$ is a constant. In liquids, $Q \gg A$ and the fluorescence quantum yield can be written:

$\eta=\frac{A}{k q_{0}[M]} \exp \left(E_{a} / k T\right)$

The absorption coefficient $\varepsilon_{0}=k_{\varepsilon} f_{B}(T)$ is proportional to the Boltzmann fractional population of the molecules $f_{B}$ in the absorbing state (Hiller and Hanson 1990). In the absence of detailed information regarding the Boltzmann fraction, it is assumed that it follows:

$f_{B}=f_{B, 0} \exp (-\Delta E / k T)$

where $f_{B, 0}$ is a constant and $\Delta E$ is related to the gap in energy between the exited state and the ground state levels. Using Eqs. 2-5, the temperature dependence of the fluorescence signal detected at a wavelength $\lambda$ can be expressed as:

$I_{f}(\lambda)=K_{\mathrm{opt}}(\lambda) K_{\mathrm{spec}}(\lambda) I_{0} c V \exp \left(\frac{\beta(\lambda)}{T}\right)$,

where $K_{\text {spec }}(\lambda)=\frac{A}{k q_{0}[M]} k_{\varepsilon} f_{B, 0} \quad$ and $\quad \beta(\lambda)=\left(E_{a}-\Delta E\right) / k$. $K_{\text {opt }}(\lambda), \quad K_{\text {spec }}(\lambda)$ and $\beta(\lambda)$ are constants during the experiments. If $E_{a}>\Delta E, \beta(\lambda)$ is positive and the fluorescence intensity is decreasing with the temperature. However, in the case of fluorescein 27, the opposite behavior is observed in Fig. 1 which means that $E_{a}<\Delta E$.

The fluorescence spectrum of the dye tracer is generally broadband, and optical filters are used to select specific spectral bands of the fluorescence emission. For a given spectral band $i$, the fluorescence intensity $I_{f}(\lambda)$ is integrated over the wavelength range $\left[\lambda_{i, 1}, \lambda_{i, 2}\right]$ :

$I_{f, i}=\int_{\lambda_{i, 1}}^{\lambda_{i, 2}} K_{\mathrm{opt}}(\lambda) K_{\mathrm{spec}}(\lambda) I_{l} c V \exp \left(\frac{\beta(\lambda)}{T}\right) \mathrm{d} \lambda$.

Based on experimental measurements, Lavieille et al. (2004) suggest to approximate this expression as:

$I_{f, i} \approx K_{\mathrm{opt}, i} K_{\mathrm{spec}, i} I_{l} c V \exp \left(\frac{A_{i}}{T^{2}}+\frac{B_{i}}{T}+C_{i}\right)$.

$A_{i}$ and $B_{i}$ are specific to a given combination of dye, solvent, excitation wavelength, and spectral band. In contrast, $C_{i}$ depends on the exact configuration of the experimental system and can change from one measurement configuration to another. The ratio $R_{f}$ of the intensities of two different spectral bands is given by:

$R_{f}=\frac{I_{f, 1}}{I_{f, 2}}=\frac{K_{\mathrm{opt}, 1} K_{\mathrm{spec}, 1}}{K_{\mathrm{opt}, 2} K_{\mathrm{spec}, 2}} \exp \left(\frac{A}{T^{2}}+\frac{B}{T}+C\right)$,

where $X=X_{1}-X_{2}$ stands for $X=A, B$ or $C$.

When the technique is applied in imagery, only $A$ and $B$ do not depend on the pixel position in the image. All other variables can change from one pixel to the other, especially the parameters $K_{\mathrm{opt}, i}, K_{\mathrm{spec}, i}$, and $C$ that are function of the arrangement of the detection system. Even under isothermal conditions, the fluorescence ratio $R_{f}$ is not necessarily uniform. To eliminate the influence of the detection system, a reference measurement at a known temperature $T_{0}$ (with the same optical configuration as for the measurement) is recorded. Denoting $R_{0}$ the fluorescence ratio obtained in the reference measurement,

$\ln \left(\frac{R_{f}}{R_{0}}\right)=A\left(\frac{1}{T^{2}}-\frac{1}{T_{0}^{2}}\right)+B\left(\frac{1}{T}-\frac{1}{T_{0}}\right)$.

When $A$ and $B$ are known from a calibration experiment (see Sect. 3.5), Eq. 10 can be used to determine the liquid temperature. However, it should be noted that its use is valid only if the fluorescence reabsorption can be neglected on the optical path from the measurement volume in the laser sheet to the surface of the detectors as required for Eq. 2. This condition will be satisfied in the measurements presented later, due to a limited dye concentration 
( $\mathrm{c}=10^{-5} \mathrm{~mol} / \mathrm{L}$ ) and the fact that droplet size is not exceeding 200 microns in the experiments. Assuming an optical path equal to $100 \mu \mathrm{m}$, reabsorption of the signal on the $505-515 \mathrm{~nm}$ band is around $1 \%$.

\subsection{Selection of the fluorescent tracer and the spectral bands}

Rhodamine B or Kiton red were among the most widely used tracer dye because of their high sensitivity to temperature and to their complete solubility in aqueous solutions (Coppeta and Rogers 1998). However, none of these tracers has been selected for this study. Instead, experiments were performed using fluorescein 27 (FL27, $\mathrm{C}_{20} \mathrm{H}_{10} \mathrm{O}_{5} \mathrm{Cl}$ ) also called fluorescein 548. This choice is based on the recent work of Sutton et al. (2008) who made a comprehensive description of the interesting features of FL27 for temperature measurements in aqueous solutions. They showed that FL27 has a temperature sensitivity significantly higher than traditional tracers including rhodamine $\mathrm{B}$ and kiton $\operatorname{red}\left(3.5 \%{ }^{\circ} \mathrm{C}\right.$ against $-1.6 \% /{ }^{\circ} \mathrm{C}$ ) for an excitation wavelength of $532 \mathrm{~nm}$, also in use in this study. However, these observations refer to the whole fluorescence spectrum, and further investigations were required to determine two spectral bands of detection for the two-color/single-dye approach.

Preliminary measurements using a spectrometer were performed in a temperature-controlled cell using the same methodology as Deprédurand et al. (2008). Figure 2 shows the absorption and emission spectra of FL27 at different temperatures. It can be noticed that the fluorescence signal increases with temperature. This behavior has been interpreted as an effect related to the increase in the absorption coefficient $\varepsilon_{0}$ at the laser wavelength with the temperature. The absorption coefficient of FL27 is approximately multiplied by 3 when the temperature increases from $20^{\circ} \mathrm{C}$ to $80^{\circ} \mathrm{C}$ (Sutton, et al. 2008). In Figure 2, it can be also noticed that a significant portion of the emission spectrum of FL27 is anti-Stokes-shifted with respect to the excitation line, i.e., it is shifted to shorter wavelengths than $532 \mathrm{~nm}$.

However, a weak point for the present application is that the absorption cross-section of FL27 is very low at $532 \mathrm{~nm}$ compared with other possible excitation wavelengths (Sutton et al. (2008). From Fig. 2, the evolution of the temperature sensitivity $\beta(\lambda)$ was calculated using Eq. 6 and the results are displayed in Fig. 3. The selected bands for temperature measurements correspond to the ranges (505$515 \mathrm{~nm})$ and $(570-600 \mathrm{~nm})$. This selection has been carried out with regards to the intensity level as well as the sensitivity to the temperature since these bands exhibit very different values of $\beta(\lambda)$.

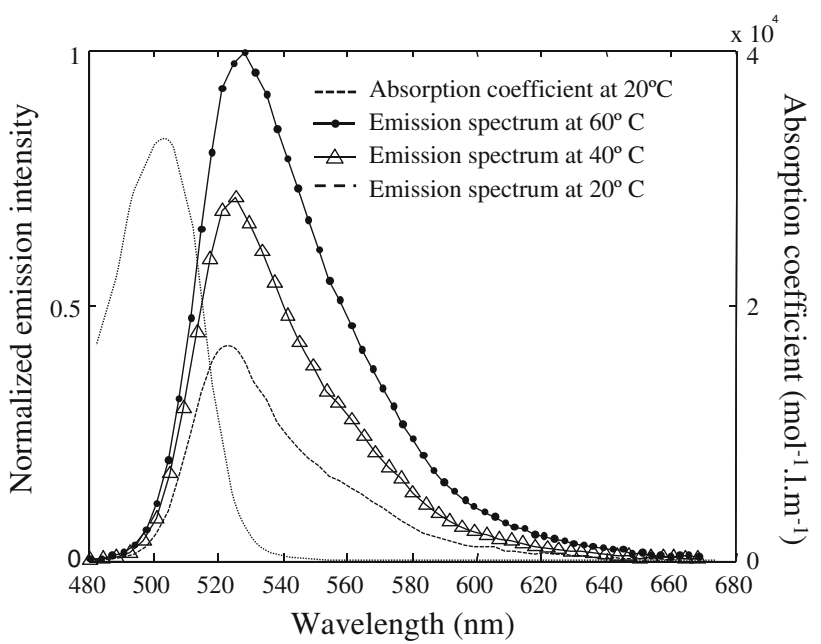

Fig. 2 The absorption coefficient and relatives emission spectra of FL27 dissolved into water as a function of the wavelength

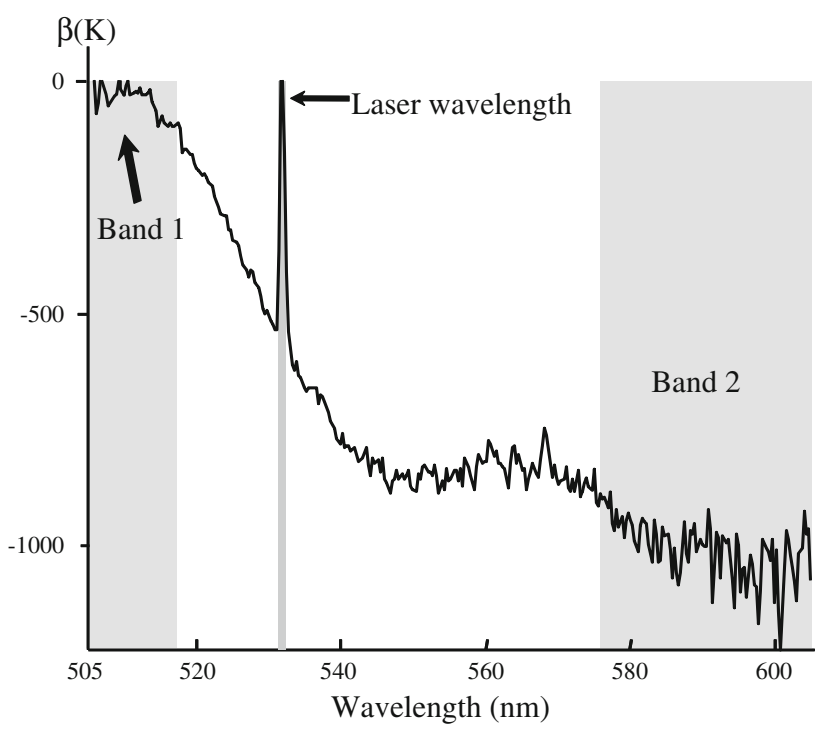

Fig. 3 The sensitivity $\beta(\lambda)$ to temperature variations of the fluorescence signal as defined in Eq. 1 and the positions of the spectral bands selected for the temperature measurement

\subsection{PLIF measurement system}

The measurement system is illustrated in Fig. 4. The excitation of FL27 is achieved by means of a CW Nd:YAG laser (Laser Quantum Ventus, $P_{\max }=1.5 \mathrm{~W}$ at $532 \mathrm{~nm}$ ). An arrangement of spherical and cylindrical lenses provides a laser sheet with a thickness of $220 \mu \mathrm{m}$ and a height of $1.6 \mathrm{~mm}$ in the measurement zone. This latter is observed by a Questar QM-1 long distance microscope which is positioned at right angle at a working distance of about $84 \mathrm{~cm}$. The microscope field of view is then about $3 \times 3 \mathrm{~mm}^{2}$. A holographic filter (Notch Plus, Kayser Optical) is used to block the Mie scattering of the laser 


$$
I(\lambda)=K_{\text {opt }}(\lambda) \varepsilon_{0} I_{0} c V \eta \exp \left(-c\left(\varepsilon_{0} a+\varepsilon(\lambda) b\right)\right),
$$

where $K_{\text {opt }}$ is a parameter depending on the optical properties of the detection system (e.g., the solid angle of the detection and the spectral sensitivity of the detectors). $c$ is the concentration in dye molecules and the product $c . V$ corresponds to the number of molecules that are illuminated by the laser beam while in the field of view of the detector. $I_{0}$ is the intensity of the laser beam before crossing the absorbing medium. The laser intensity in the absorbing medium is affected by the absorption of the incident laser beam, and the fluorescence emission can be partially reabsorbed. $a$ and $b$ denote the optical path length of the laser beam and the fluorescence in the liquid medium. $\varepsilon_{0}$ is the coefficient of absorption at the wavelength of the laser and $\varepsilon(\lambda)$ at a given wavelength of the fluorescence spectrum. If absorption can be neglected, Eq. 1 simplifies into:

$I(\lambda)=K_{\text {opt }}(\lambda) \varepsilon_{0} I_{0} c V \eta$.

The temperature dependence of the fluorescent emission appears on the one hand in the absorption coefficient $\varepsilon_{0}$ and on the other hand in the fluorescence quantum yield $\eta$. The fluorescence quantum yield is defined by $\eta=A / A+Q$ where $A$ is the Einstein coefficient for spontaneous emission and $Q$ the electronic quenching rate. The quenching rate is proportional to the concentration in quenchers $[M]$, which is assumed to be constant. According to Glowacki (1964), the quenching rate can be given by:

$Q=k q_{0}[M] \exp \left(-E_{a} / k T\right)$.

where $E_{a}$ is the activation energy involved in collisions between a fluorescent molecule and a molecule of the solvent and $k q_{0}$ is a constant. In liquids, $Q \gg A$ and the fluorescence quantum yield can be written:

$\eta=\frac{A}{k q_{0}[M]} \exp \left(E_{a} / k T\right)$

The absorption coefficient $\varepsilon_{0}=k_{\varepsilon} f_{B}(T)$ is proportional to the Boltzmann fractional population of the molecules $f_{B}$ in the absorbing state (Hiller and Hanson 1990). In the absence of detailed information regarding the Boltzmann fraction, it is assumed that it follows:

$f_{B}=f_{B, 0} \exp (-\Delta E / k T)$

where $f_{B, 0}$ is a constant and $\Delta E$ is related to the gap in energy between the exited state and the ground state levels. Using Eqs. 2-5, the temperature dependence of the fluorescence signal detected at a wavelength $\lambda$ can be expressed as:

$I_{f}(\lambda)=K_{\mathrm{opt}}(\lambda) K_{\mathrm{spec}}(\lambda) I_{0} c V \exp \left(\frac{\beta(\lambda)}{T}\right)$,

where $K_{\text {spec }}(\lambda)=\frac{A}{k q_{0}[M]} k_{\varepsilon} f_{B, 0} \quad$ and $\quad \beta(\lambda)=\left(E_{a}-\Delta E\right) / k$. $K_{\text {opt }}(\lambda), \quad K_{\text {spec }}(\lambda)$ and $\beta(\lambda)$ are constants during the experiments. If $E_{a}>\Delta E, \beta(\lambda)$ is positive and the fluorescence intensity is decreasing with the temperature. However, in the case of fluorescein 27, the opposite behavior is observed in Fig. 1 which means that $E_{a}<\Delta E$.

The fluorescence spectrum of the dye tracer is generally broadband, and optical filters are used to select specific spectral bands of the fluorescence emission. For a given spectral band $i$, the fluorescence intensity $I_{f}(\lambda)$ is integrated over the wavelength range $\left[\lambda_{i, 1}, \lambda_{i, 2}\right]$ :

$I_{f, i}=\int_{\lambda_{i, 1}}^{\lambda_{i, 2}} K_{\mathrm{opt}}(\lambda) K_{\mathrm{spec}}(\lambda) I_{l} c V \exp \left(\frac{\beta(\lambda)}{T}\right) \mathrm{d} \lambda$.

Based on experimental measurements, Lavieille et al. (2004) suggest to approximate this expression as:

$I_{f, i} \approx K_{\mathrm{opt}, i} K_{\mathrm{spec}, i} I_{l} c V \exp \left(\frac{A_{i}}{T^{2}}+\frac{B_{i}}{T}+C_{i}\right)$.

$A_{i}$ and $B_{i}$ are specific to a given combination of dye, solvent, excitation wavelength, and spectral band. In contrast, $C_{i}$ depends on the exact configuration of the experimental system and can change from one measurement configuration to another. The ratio $R_{f}$ of the intensities of two different spectral bands is given by:

$R_{f}=\frac{I_{f, 1}}{I_{f, 2}}=\frac{K_{\mathrm{opt}, 1} K_{\mathrm{spec}, 1}}{K_{\mathrm{opt}, 2} K_{\mathrm{spec}, 2}} \exp \left(\frac{A}{T^{2}}+\frac{B}{T}+C\right)$,

where $X=X_{1}-X_{2}$ stands for $X=A, B$ or $C$.

When the technique is applied in imagery, only $A$ and $B$ do not depend on the pixel position in the image. All other variables can change from one pixel to the other, especially the parameters $K_{\mathrm{opt}, i}, K_{\mathrm{spec}, i}$, and $C$ that are function of the arrangement of the detection system. Even under isothermal conditions, the fluorescence ratio $R_{f}$ is not necessarily uniform. To eliminate the influence of the detection system, a reference measurement at a known temperature $T_{0}$ (with the same optical configuration as for the measurement) is recorded. Denoting $R_{0}$ the fluorescence ratio obtained in the reference measurement,

$\ln \left(\frac{R_{f}}{R_{0}}\right)=A\left(\frac{1}{T^{2}}-\frac{1}{T_{0}^{2}}\right)+B\left(\frac{1}{T}-\frac{1}{T_{0}}\right)$.

When $A$ and $B$ are known from a calibration experiment (see Sect. 3.5), Eq. 10 can be used to determine the liquid temperature. However, it should be noted that its use is valid only if the fluorescence reabsorption can be neglected on the optical path from the measurement volume in the laser sheet to the surface of the detectors as required for Eq. 2. This condition will be satisfied in the measurements presented later, due to a limited dye concentration 
( $\mathrm{c}=10^{-5} \mathrm{~mol} / \mathrm{L}$ ) and the fact that droplet size is not exceeding 200 microns in the experiments. Assuming an optical path equal to $100 \mu \mathrm{m}$, reabsorption of the signal on the $505-515 \mathrm{~nm}$ band is around $1 \%$.

\subsection{Selection of the fluorescent tracer and the spectral bands}

Rhodamine B or Kiton red were among the most widely used tracer dye because of their high sensitivity to temperature and to their complete solubility in aqueous solutions (Coppeta and Rogers 1998). However, none of these tracers has been selected for this study. Instead, experiments were performed using fluorescein 27 (FL27, $\mathrm{C}_{20} \mathrm{H}_{10} \mathrm{O}_{5} \mathrm{Cl}$ ) also called fluorescein 548. This choice is based on the recent work of Sutton et al. (2008) who made a comprehensive description of the interesting features of FL27 for temperature measurements in aqueous solutions. They showed that FL27 has a temperature sensitivity significantly higher than traditional tracers including rhodamine $\mathrm{B}$ and kiton $\operatorname{red}\left(3.5 \%{ }^{\circ} \mathrm{C}\right.$ against $-1.6 \% /{ }^{\circ} \mathrm{C}$ ) for an excitation wavelength of $532 \mathrm{~nm}$, also in use in this study. However, these observations refer to the whole fluorescence spectrum, and further investigations were required to determine two spectral bands of detection for the two-color/single-dye approach.

Preliminary measurements using a spectrometer were performed in a temperature-controlled cell using the same methodology as Deprédurand et al. (2008). Figure 2 shows the absorption and emission spectra of FL27 at different temperatures. It can be noticed that the fluorescence signal increases with temperature. This behavior has been interpreted as an effect related to the increase in the absorption coefficient $\varepsilon_{0}$ at the laser wavelength with the temperature. The absorption coefficient of FL27 is approximately multiplied by 3 when the temperature increases from $20^{\circ} \mathrm{C}$ to $80^{\circ} \mathrm{C}$ (Sutton, et al. 2008). In Figure 2, it can be also noticed that a significant portion of the emission spectrum of FL27 is anti-Stokes-shifted with respect to the excitation line, i.e., it is shifted to shorter wavelengths than $532 \mathrm{~nm}$.

However, a weak point for the present application is that the absorption cross-section of FL27 is very low at $532 \mathrm{~nm}$ compared with other possible excitation wavelengths (Sutton et al. (2008). From Fig. 2, the evolution of the temperature sensitivity $\beta(\lambda)$ was calculated using Eq. 6 and the results are displayed in Fig. 3. The selected bands for temperature measurements correspond to the ranges (505$515 \mathrm{~nm})$ and $(570-600 \mathrm{~nm})$. This selection has been carried out with regards to the intensity level as well as the sensitivity to the temperature since these bands exhibit very different values of $\beta(\lambda)$.

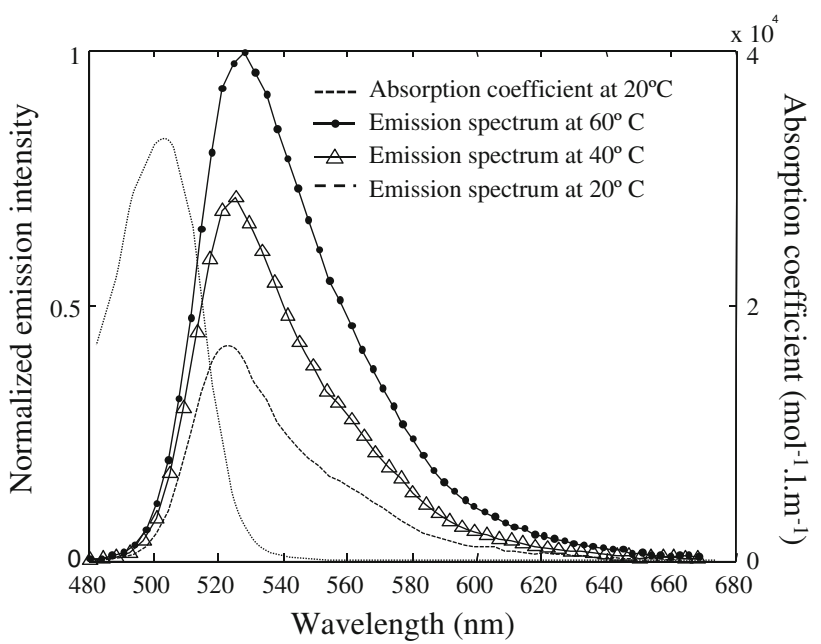

Fig. 2 The absorption coefficient and relatives emission spectra of FL27 dissolved into water as a function of the wavelength

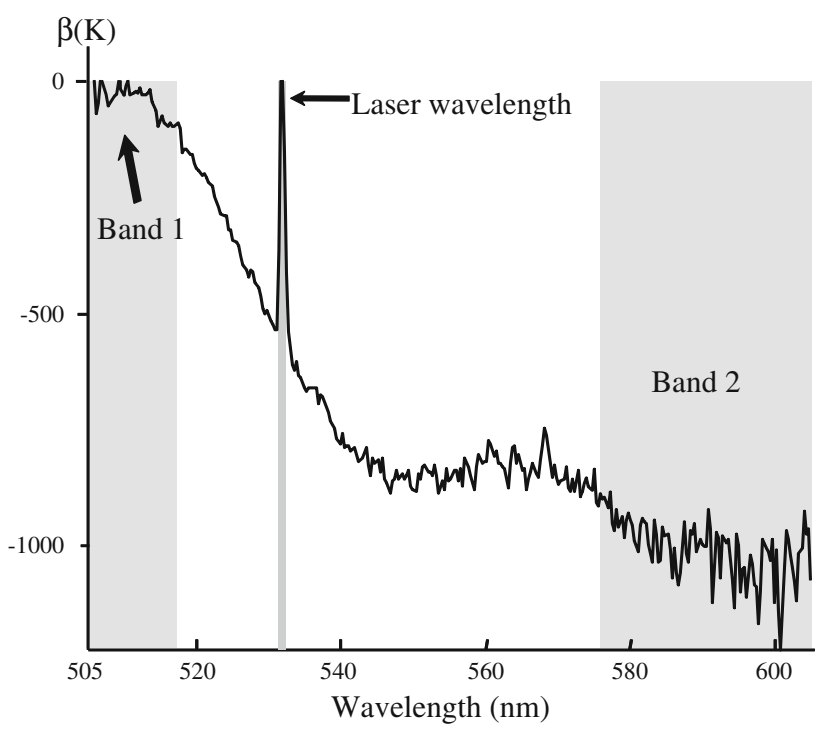

Fig. 3 The sensitivity $\beta(\lambda)$ to temperature variations of the fluorescence signal as defined in Eq. 1 and the positions of the spectral bands selected for the temperature measurement

\subsection{PLIF measurement system}

The measurement system is illustrated in Fig. 4. The excitation of FL27 is achieved by means of a CW Nd:YAG laser (Laser Quantum Ventus, $P_{\max }=1.5 \mathrm{~W}$ at $532 \mathrm{~nm}$ ). An arrangement of spherical and cylindrical lenses provides a laser sheet with a thickness of $220 \mu \mathrm{m}$ and a height of $1.6 \mathrm{~mm}$ in the measurement zone. This latter is observed by a Questar QM-1 long distance microscope which is positioned at right angle at a working distance of about $84 \mathrm{~cm}$. The microscope field of view is then about $3 \times 3 \mathrm{~mm}^{2}$. A holographic filter (Notch Plus, Kayser Optical) is used to block the Mie scattering of the laser 


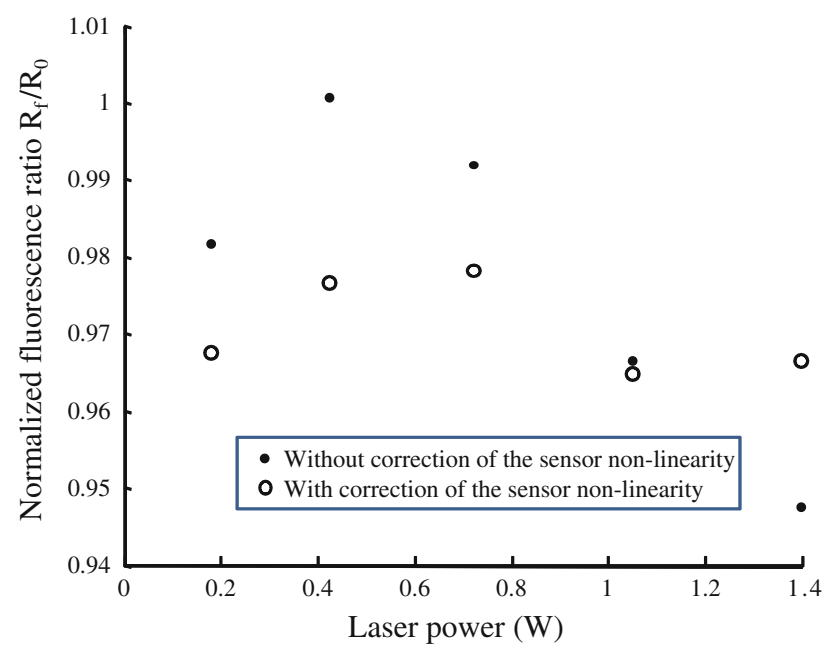

Fig. 8 Evolution of the normalized fluorescence ratio as a function of the laser power in the case of an isothermal droplet stream

It allows determining the physical coordinates of each point in the image. In these works, the field of view of the cameras was much larger than in the present study and an equally spaced grid pattern at a few millimeter intervals can be used. In the present case, the same method is difficult to adapt in regard to the spatial resolution: 1 pixel correspond to about $12 \mu \mathrm{m}$ with a $4 \times 4$ binning. For this reason, another procedure has been used for the pixel correspondence. It consists in finding the translation and the rotation to apply to the image of one camera to have the best match with the image of the other camera. Introducing the row and column position $\left(x_{1, i}, y_{1, i}\right)$ of the $i$ th pixel in the image of the 1 st camera, the pixel is moved to the position $\left(x_{2, i}, y_{2, i}\right)$ using the following transformation:

$\left(\begin{array}{l}x_{2, i} \\ y_{2, i}\end{array}\right)=\left(\begin{array}{cc}\cos \theta & \sin \theta \\ -\sin \theta & \cos \theta\end{array}\right)\left(\begin{array}{l}x_{1, i} \\ y_{1, i}\end{array}\right)+\left(\begin{array}{l}t_{x} \\ t_{y}\end{array}\right)$,

where $\theta$ is the rotation angle and $t_{x}$ and $t_{y}$ the Cartesian components of the translation. Possible distortions are not taken into account in Eq. 12 in contrast to Sakakibara and Adrian (2004). The risk of a difference in magnification is extremely limited in the present setup because the cameras are placed behind the same microscope. To avoid off-plane displacements, a grid of points is positioned at $45^{\circ}$ in front of the microscope. The position of the cameras is adjusted to have the focus in the same central zone of the image. However, using this grid to get the optimal rotation and translation is not sufficient to reach a subpixel accuracy in the positioning.

The best match between the images is found when minimizing the distance $S\left(\theta, t_{x}, t_{y}\right)$ between the images defined as:

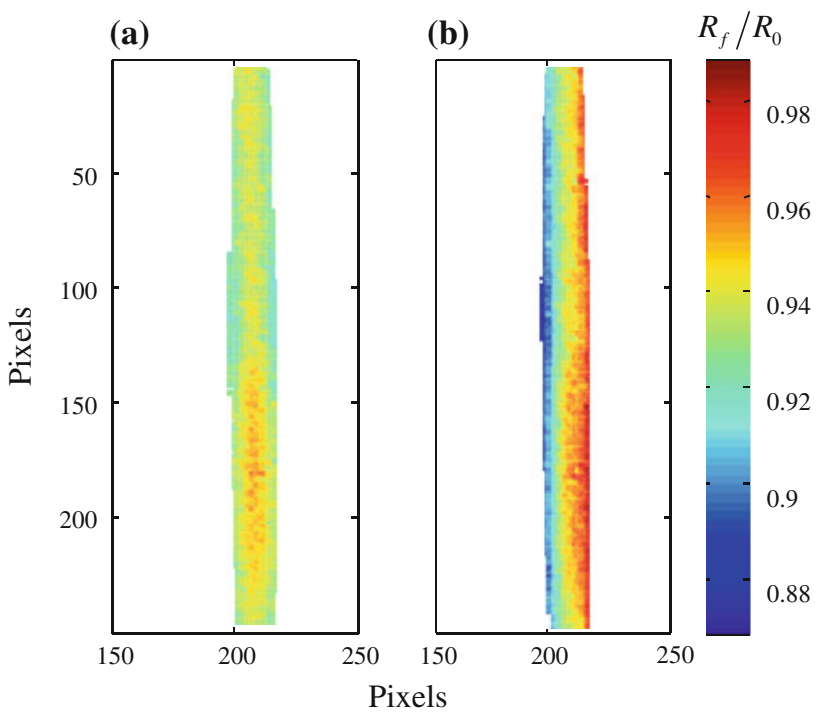

Fig. 9 Sensitivity of the normalized fluorescence ratio $R_{f} / R_{0}$ to the triplet $\quad\left(\theta, t_{x}, t_{y}\right), \quad$ a $\quad \theta=-0.30^{\circ}, t_{x}=0.36, t_{y}=6.84, \quad$ b $\theta=-0.30^{\circ}, t_{x}=0.86, t_{y}=6.84$

$S\left(\theta, t_{x}, t_{y}\right)=\frac{1}{N} \sqrt{\sum_{i_{1}=1}^{N}\left[I_{1}\left(x_{1, i}, y_{1, i}\right) / I_{1, \max }-\widehat{I}_{2}\left(x_{2, i}, y_{2, i}\right) / I_{2, \max }\right]^{2}}$.

In this expression, $I_{1}$ and $I_{2}$ denote the images of the cameras subtracted of their dark noise, while $I_{1, \max }$ and $I_{2, \max }$ correspond to their maximum intensities and $N$ is the number of pixels in the images. Since $x_{2, i}$ and $y_{2, i}$ are not necessarily integers, $\widehat{I}_{2}\left(x_{2, i}, y_{2, i}\right)$ is obtained by a bilinear interpolation. Classical minimization algorithms are used to achieve a subpixel convergence of $\left(x_{2, i}, y_{2, i}\right)$. The convergence error for $\left(x_{2, i}, y_{2, i}\right)$ is typically in the order of 0.1 pixel, but this may depend on the distribution of the intensity field in the image. Figure $9 \mathrm{a}$ shows the distribution of the fluorescence ratio $R_{f} / R_{0}$ in an isothermal droplet stream after convergence of $\left(\theta, t_{x}, t_{y}\right)$. As expected, the fluorescence ratio is almost uniform. In contrast, in Fig. 9b, a small variation of 0.5 pixel in $t_{x}$ leads to an important disruption in the distribution of the fluorescence ratio with a strong gradient in the $x$-direction.

\subsection{Temperature calibration}

The temperature calibration is carried out in a cell filled with an aqueous solution of FL27. The liquid temperature is increased by means of an immersed heater. Isothermal conditions are maintained throughout the experiments by stirring the liquids. The solution has a moderate concentration in FL27 ( $\left.c=5 \times 10^{-7} \mathrm{~mol} / \mathrm{L}\right)$, and the optical path between the laser sheet and the cell window does not exceed $1 \mathrm{~mm}$ to limit as much as possible the reabsorption 


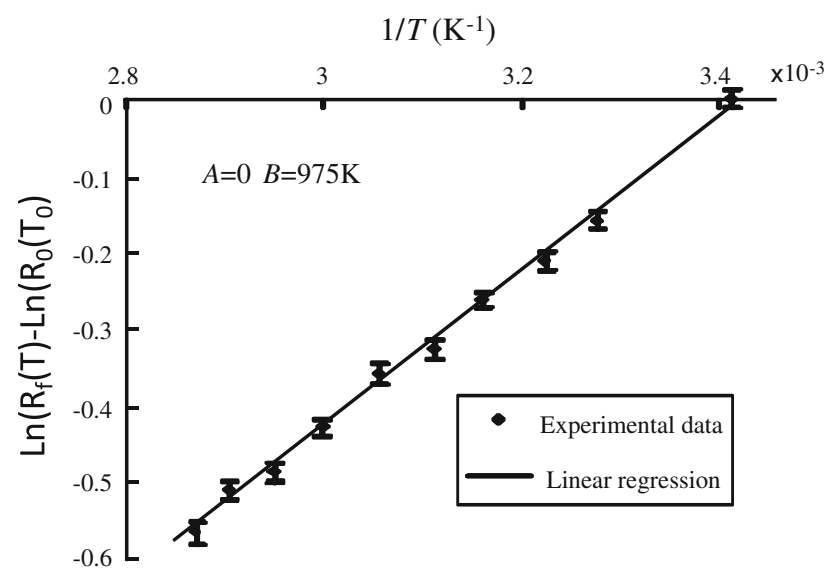

Fig. 10 Temperature calibration curves and the temperature standard deviation corresponding to the images

of the fluorescence in the first spectral band of detection. The images are first subtracted from the background noise, and then, they are corrected for the non-linearity of the optical system (see Sect. 3.3). Finally, the procedure described in Sect. 3.4 is applied to ensure their correspondence and the fluorescence ratio $R_{f}$ is calculated. An almost linear trend is observed for $\ln \left(R_{f}(1 / T)\right)-$ $\ln \left(R_{0}\left(1 / T_{0}\right)\right)$ as a function of $1 / T$ (Fig. 10). The slope corresponds to a mean variation in the fluorescence ratio of about $0.8 \% /{ }^{\circ} \mathrm{C}$ in the temperature range $\left[20^{\circ} \mathrm{C}-75^{\circ} \mathrm{C}\right]$. From Fig. 10, the coefficients $A$ and $B$ in Eq. 9 can be determined. The standard deviation is also displayed with error bars in Fig. 10, and it corresponds to an uncertainty of about \pm 0.008 on $R_{f}$. Therefore, the measurement errors related to the calibration are not expected to exceed $\pm 1^{\circ} \mathrm{C}$ on the temperature. This sensitivity of fluorescence ratio (about $0.8 \% /{ }^{\circ} \mathrm{C}$ ) appears sufficient for the measurements, but it is much smaller than the sensitivity reported by Sutton et al.(2008) with a two-dye two-color approach with a couple FL27/Kiton red (as high as $7 \% /{ }^{\circ} \mathrm{C}$ in the range $20^{\circ} \mathrm{C}-80^{\circ} \mathrm{C}$ ). However, the use of two dyes can have some problems. The ratio of the concentration of the tracers must be kept constant during the measurements and the calibration of the system. Measurements require a careful calibration due to the potential overlay between the emission spectra of both dyes.

\section{Validation tests in temperature-controlled conditions}

\subsection{Isothermal droplet streams}

The first validation test was to measure the fluorescence ratio of a droplet stream injected at room temperature. Figure 11 shows the superimposition of the images of the fluorescence ratio obtained by moving the droplet stream in

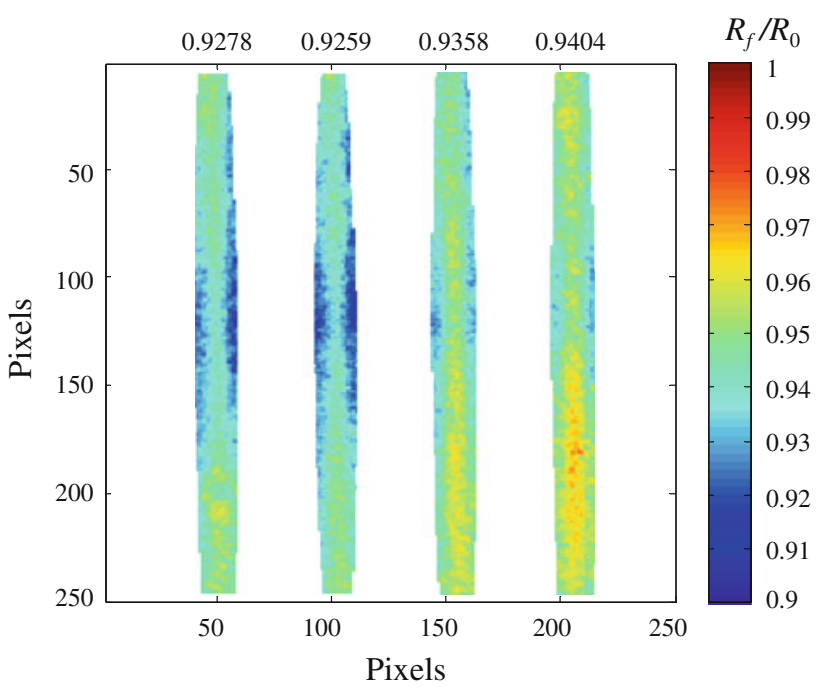

Fig. 11 Fluorescence ratio $R_{f}$ for different positions of the droplets stream in the image scene. Normalization by the fluorescence ratio $R_{0}$ measured in a cell at the same temperature $T=19^{\circ} \mathrm{C}$ (average of 150 images). Values at the top of the image correspond to the average values of $R_{f} / R_{0}$ in the streams

the $x$-direction at 4 different positions. In this figure, the fluorescence ratio $R_{f}$ is normalized by the ratio $R_{0}$ measured in a cell at the same temperature. The fluorescence ratio is thus expected to be identical regardless of the position of the droplet stream. However, there are small variations within the droplet streams and between the droplets streams. These variations reach about $2 \%$ and they cannot be explained simply by a lack of statistical convergence due to the measurement noise. The measurement ratio on the stream edge may be relatively inaccurate as indicated in Fig. 12 which represents the uncertainty of the convergence of the fluorescence ratio when averaging over 150 images but a reasonable accuracy can be achieved in the center of the droplet streams where the uncertainty of the convergence is less than $1 \%$.

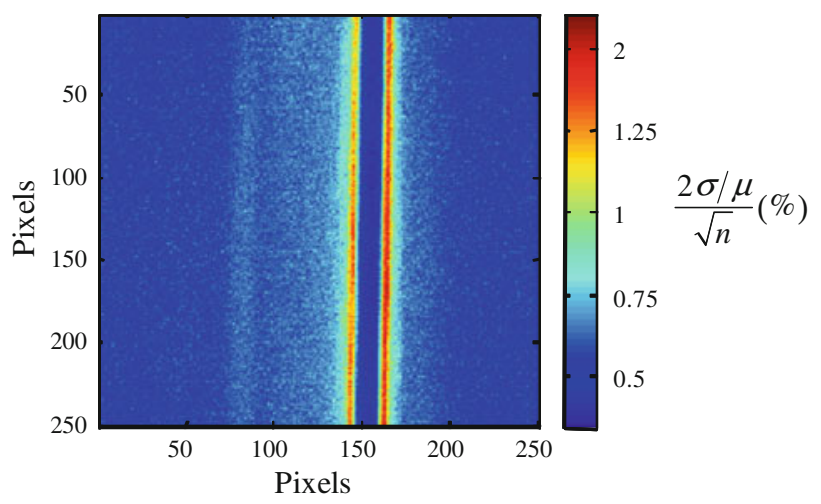

Fig. 12 Image of the uncertainty of the convergence of the fluorescence ratio $\mathrm{R}_{\mathrm{f}}(\sigma$ : its standard deviation, $\mu$ : its average, $n=150$ the number of cumulated images) 


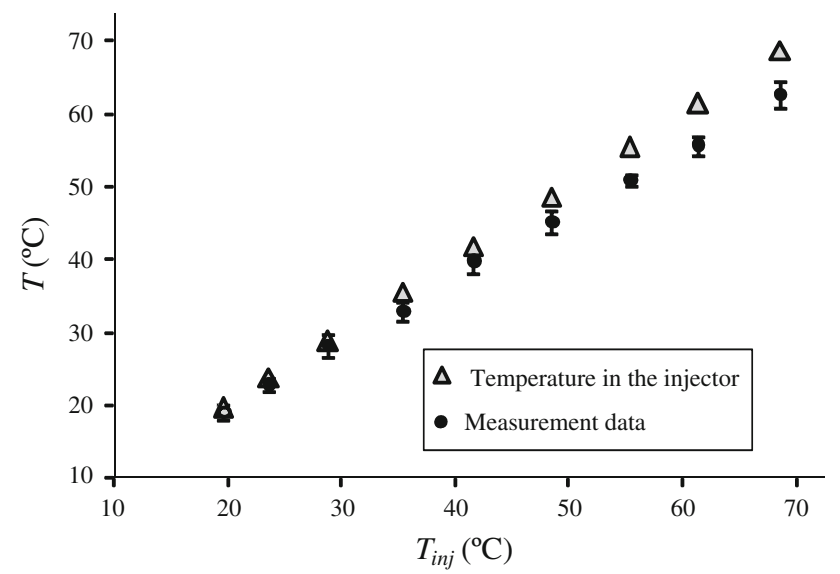

Fig. 13 Evolution of the average temperature measured in the droplet stream as a function of the liquid temperature in the injector and its standard deviation in the images of the temperature field as error bars

These variations in $R_{f} / R_{0}$ when this ratio should be uniform are probably related to optical aberrations that are rather large as pointed out in Fig. 6. In the present optical system, these aberrations are partly due to the increased reflection of light rays when they strike the mirror of the long distance microscope near its edge, in comparison with those that strike nearer its centre (in such a long distance microscope similar to a Cassegrain telescope in its arrangement, paraxial rays which lies close to the optical axis are stopped). The effect of these aberrations in an image depends on the light distribution in the observed area. It is more visible in an image of the cell (where the field of view is fully illuminated by the laser sheet) than in the case of a thin droplet stream. The elimination of the parameters $K_{\mathrm{opt}, 1}$ and $K_{\mathrm{opt}, 2}$ when doing the ratio $R_{f} / R_{0}$ to obtain Eq. 5 is therefore no longer fully justified. Removing the optical aberrations remains for the moment something difficult and it will be considered that the errors generated in this way are in the order of $2 \%$ on the fluorescence ratio.

Figure 11 also reveals that the normalized fluorescence ratio is about 0.93 in the droplet streams while the temperature is the same than in the cell. This could be explained by an effect related to the droplet curvature that has already been described for other tracers. Labergue et al. (2010) showed that the fluorescence ratio can be influenced by the droplet size. This effect was reported for kiton red and pyrromethene 597-8C9 especially for small droplet sizes typically below $80 \mu \mathrm{m}$, but FL27 was not investigated. The study of this phenomenon is beyond the scope of the present article and is not accounted for in Sect. 4.1. In the following, the fluorescence ratio $R_{f}(T)$ will continue to be divided by the reference ratio $R_{0}\left(T_{0}\right)$ measured in a cell, but to minimize the errors due this effect $R_{f}(T) / R_{0}\left(T_{0}\right)$ in Eq. 5 will be multiplied by the factor $R_{0}\left(T_{0}\right) / R_{f}\left(T_{0}\right)$ where $R_{f}\left(T_{0}\right)$ is measured on droplets near the injector exit at the temperature $T_{0}$. In addition, a relatively high concentration of tracer $\left(c=10^{-5} \mathrm{~mol} / \mathrm{L}\right)$ will be used to ensure a sufficient level of fluorescence signal and limit this effect as well (Labergue et al. 2010).

\subsection{Heated droplet streams}

The temperature field was also measured at a distance of $4 \mathrm{~cm}$ from the injector exit while the temperature of the liquid in the injector is increased from $19^{\circ} \mathrm{C}$ to $68^{\circ} \mathrm{C}$. This distance was chosen to ensure that the measurement zone is located after the break up of the liquid jet, whatever the injection temperature. Figure 13 presents the average temperature in the droplet stream as a function of the liquid temperature in the injector $T_{\text {inj }}$ measured by a thermocouple. Each point in the figure corresponds to the spatial averaging of the temperature in the pixels situated in the droplet stream. Measurements also results from the average of 150 images.

The temperature measured in the droplet stream is slightly lower than that in the injector and the difference increases with $T_{\text {inj. }}$. This difference may be due to the droplet cooling in ambient air by convection and evaporation since these phenomena become more important as the droplet temperature increases. However, as expected, the gap remains limited due to the short distance between the injector exit and the measurement zone, and the difference reaches $5^{\circ} \mathrm{C}$ when $T_{\text {inj }}=68^{\circ} \mathrm{C}$.

\section{Application to droplets impinging onto a heated wall}

The measurement technique was finally used to characterize the temperature variation in droplets impinging onto a heated wall. Depending on the wall temperature $T_{w}$ and the normal Weber number $\mathrm{We}_{n}$ of the droplets, several regimes of impact can be observed with the HS camera: rebound, splashing, or deposition of a liquid film (Figures 14, 15, and 16a). For each of these regimes, the heat transfer between the droplet and the wall is different (Castanet et al. 2009).

In the first examples, the wall temperature is maintained above the Leidenfrost point. For an incidence angle of $22^{\circ}$, a rebound is observed (Fig. 14a) while there is a splashing for a normal incidence (Fig. 15a). In these experiments, the droplet size was adjusted to $150 \mu \mathrm{m}$ and their velocity was set to $8.2 \mathrm{~m} / \mathrm{s}$ at the injection. Figures $14 \mathrm{~b}$ and $15 \mathrm{~b}$ present the distribution of the fluorescence intensity. The mirror effect that can be observed in the images is due to the reflection of the droplet fluorescence on the polished surface of the heated wall. It can be noticed that the 
Fig. 14 Case of a rebound regime. $\left(T_{w}=400^{\circ} \mathrm{C}\right.$, $f=10552 \mathrm{~Hz}, D=150 \mu \mathrm{m}$, $V_{n}=3.1 \mathrm{~m} / \mathrm{s}, \mathrm{We}_{n}=19$, $T_{\text {inj }}=24.4^{\circ} \mathrm{C}, \alpha=22^{\circ}$ ) a image of the high-speed camera, b fluorescence intensity field, $\mathbf{c}$ temperature field measured by 2 CLIF thermography (a)
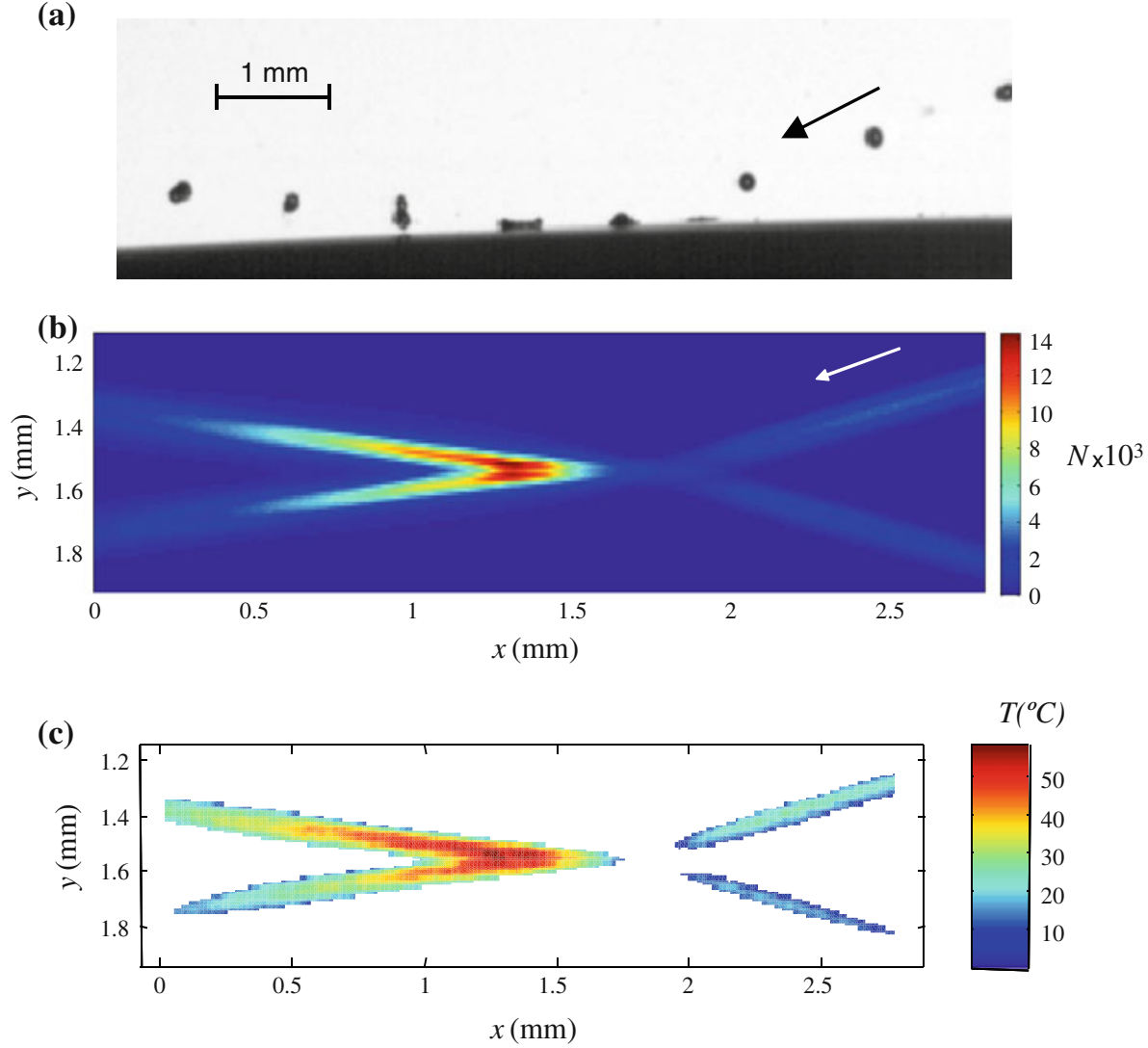

Fig. 15 Case of a normal splashing $\left(T_{w}=400^{\circ} \mathrm{C}\right.$, $f=10552 \mathrm{~Hz}, D=150 \mu \mathrm{m}$, $V_{n}=8.16 \mathrm{~m} / \mathrm{s}, \mathrm{We}_{n}=132$, $\left.T_{\mathrm{inj}}=28^{\circ} \mathrm{C}\right)$ a image of the high-speed camera,

b fluorescence intensity field, c temperature field measured by 2CLIF thermography (a)

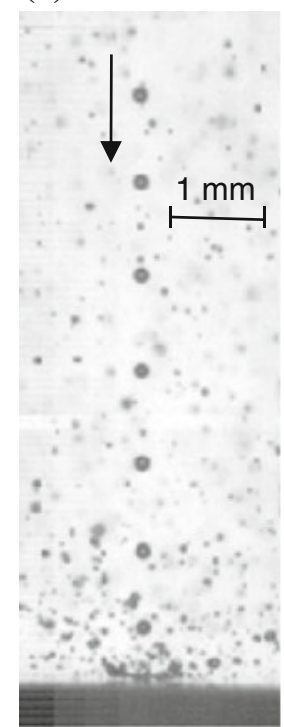

(b)

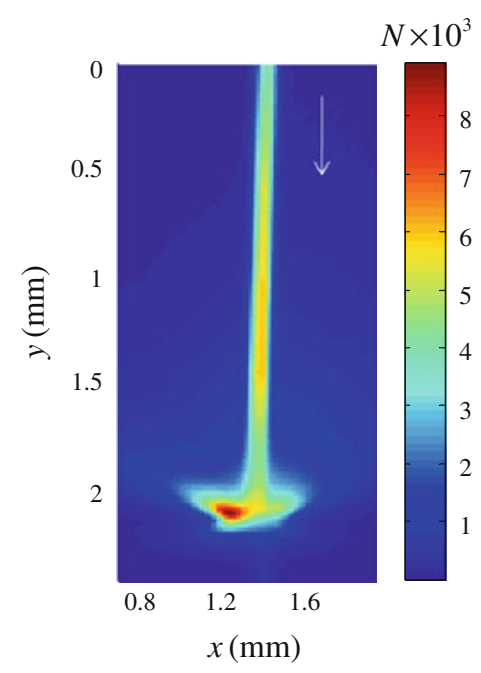

(c)

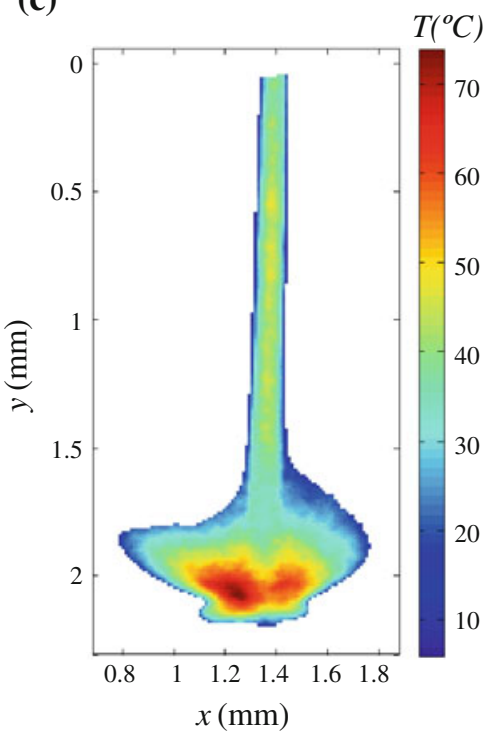

fluorescence intensity varies significantly within the images. In the case of a rebound, the vertical elongation of the droplets results in an increase in the fluorescence after the impact. Since the laser sheet is oriented vertically and has a thickness of $220 \mu \mathrm{m}$, the droplets are illuminated mostly by the central part of the Gaussian profile of the laser sheet. In contrast, when the droplets start impinging the wall, they take an oblate shape and absorb less of laser light because they tend to become wider than the laser sheet (Fig. 14b). In the case of a splashing, most of the secondary droplets leave the depth of field of the microscope and therefore becomes almost undetectable. The signal level decreases therefore rapidly with the distance to the impact zone (Fig. 15b). 
Fig. 16 Case of liquid boiling film $\left(T_{w}=200^{\circ} \mathrm{C}\right.$, $f=10552 \mathrm{~Hz}, D=150 \mu \mathrm{m}$, $V_{n}=2.5 \mathrm{~m} / \mathrm{s}, \mathrm{We}_{n}=12.4$, $T_{\text {inj }}=24.4^{\circ} \mathrm{C}$ ) $\mathbf{a}$ image of the high-speed camera,

b fluorescence intensity field, c temperature field measured by 2CLIF thermography (the rectangle in red indicate the zone where the temperature is measured) (a)

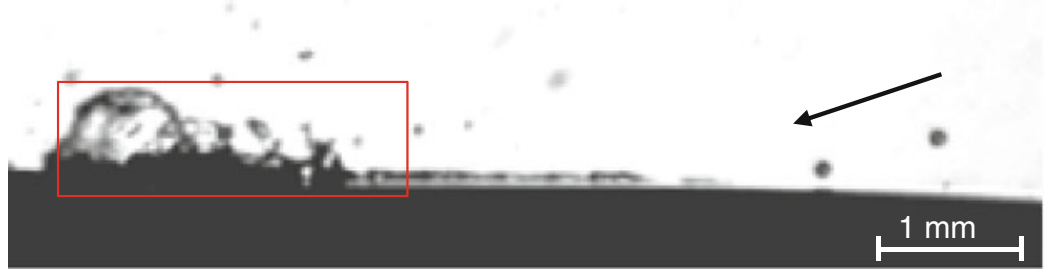

(b)

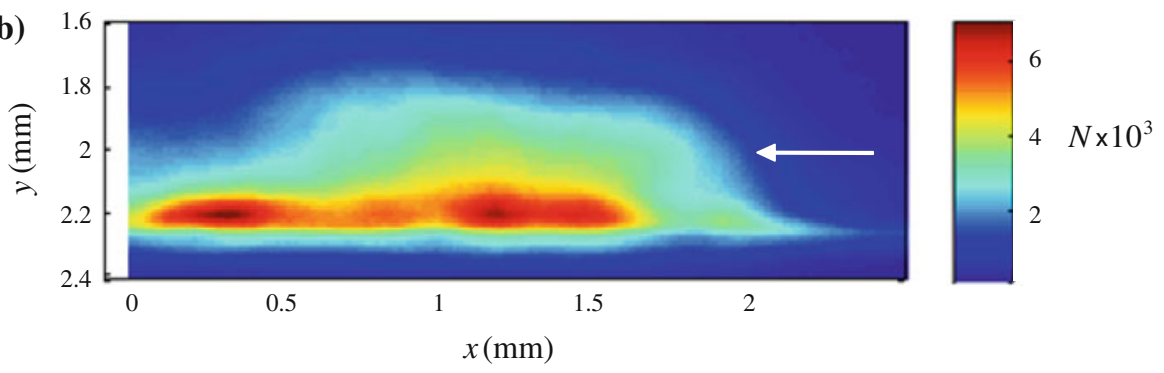

(c)
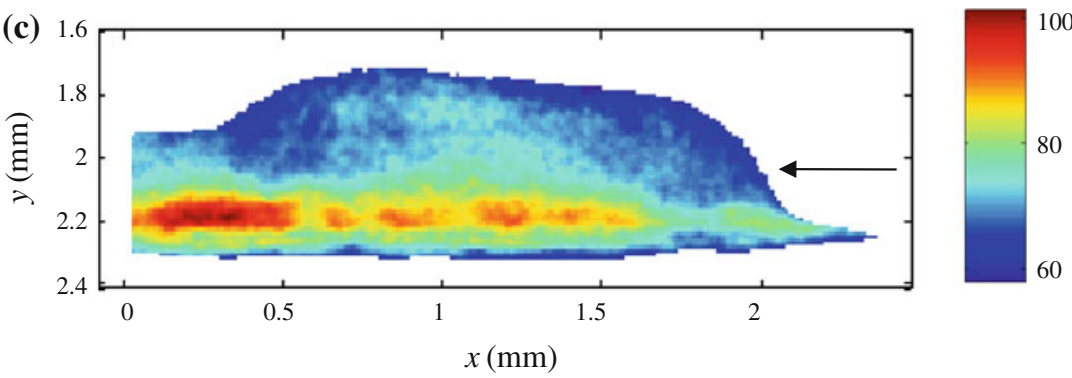

$T\left({ }^{\circ} \mathrm{C}\right)$
The corresponding temperature fields are presented in Figs. 14c and 15c. As expected, the droplet heating during the impingement appears to be larger in the case of a splashing than in the case of a rebound. The same observation was made by Castanet et al. (2009). However, the experimental conditions were not the same; in particular, the incident droplet size was significantly lower. The temperature fields in Figs. $14 \mathrm{c}$ and $15 \mathrm{c}$ are obtained by averaging intensities of 100 images. The fluorescence ratio is not defined outside the field of presence of droplets. To keep only the most significant areas in the image, pixels having a signal level lower than 1000 were eliminated in the calculation of the temperature. This threshold was chosen arbitrarily, but it is clear that the accuracy of the temperature measurement depends on the signal level. If the signal level is just above the background noise, then the fluorescence ratio will be determined with a very poor accuracy. Based on Fig. 5, it is possible to estimate the accuracy of the measured fluorescence signal. The uncertainty is given by the standard deviation $\sigma_{I}=\sigma / \sqrt{n}$, where $n=100$ is the number of images in the series and $\sigma$ can be read directly in Fig. 5 knowing the average level $\mu$ of the pixel. Finally, for the fluorescence ratio $R_{f}$, the uncertainty $\sigma_{R f}$ can be derived from:
$\frac{\sigma_{R f}}{R_{f}}=\frac{\sigma_{I_{1}}}{\mu_{1}}+\frac{\sigma_{I_{2}}}{\mu_{2}}$

where the subscripts 1 and 2 denote cameras 1 and 2 . Figure $17 \mathrm{a}, \mathrm{b}$ show the distribution of $2 \sigma_{R f} / R_{f}$ in the case of the rebound and the normal splashing. It appears that $2 \sigma_{R f} / R_{f}$ increases sharply outside the incident droplet stream and the impact zone. Inside these two regions, a reasonable accuracy is obtained for the fluorescence ratio, approximately 1 to $1.5 \%$ which roughly corresponds to a temperature uncertainty ranging from $1^{\circ} \mathrm{C}$ to $2^{\circ} \mathrm{C}$.

For a wall temperature between the boiling point and the Leidenfrost temperature of water, the film boiling regime occurs (Fig. 16a). Vapor bubbles are formed and generate secondary droplets when they burst. To study this impact regime, the droplet size and velocity were unchanged compared with the previous examples, while the wall temperature was decreased to $200^{\circ} \mathrm{C}$. Figure $16 \mathrm{~b}$, c show the fluorescence and temperature fields. The entire temperature field cannot be visible with the microscope due its limited field of view. Therefore, the emphasis was placed on observation of the area where the boiling mainly occurs, i.e., on the left-hand side of Fig. 16a. A rectangular box has been added in Fig. 16a to indicate the region corresponding 
Fig. 17 Distribution of the parameter $2 \sigma_{R f} / R_{f}$ in percent for the examples corresponding to Figs. 14, 15 and 16
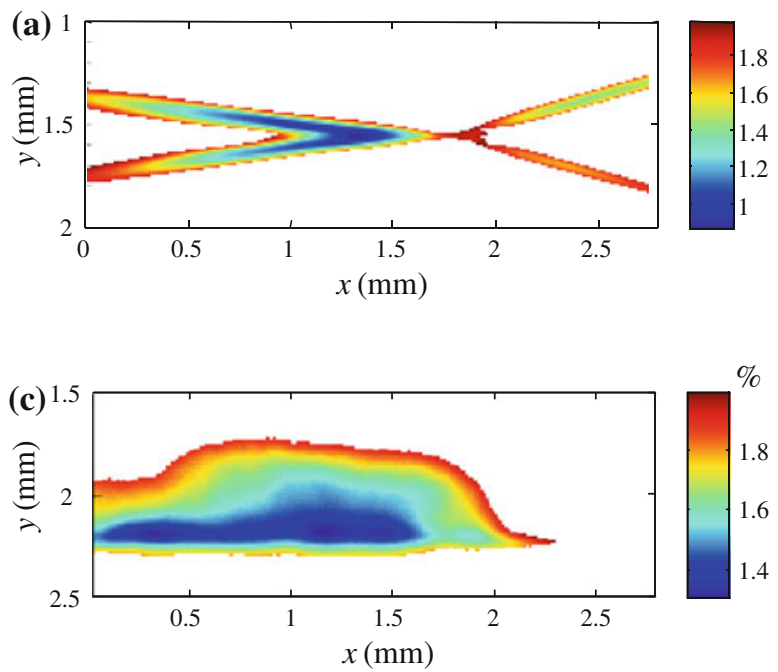

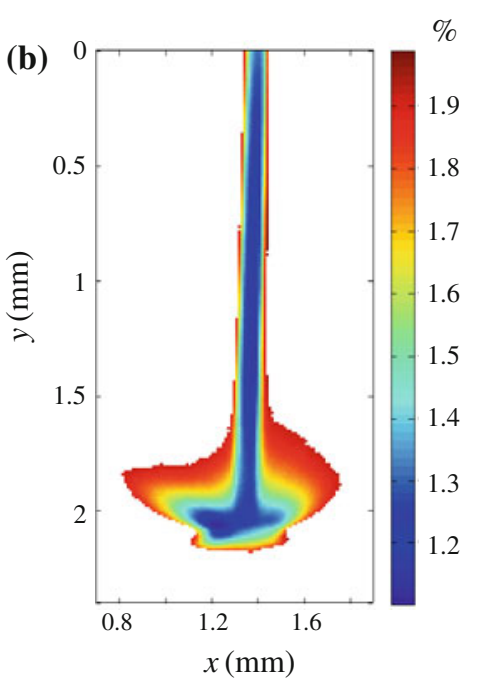

to the temperature measurement. As expected, in Fig. 16c, it can be noticed that the liquid temperature is not uniform in the liquid film.

The temperature is lower than the boiling point in the right-hand side of the picture from where the droplets arrive and it reaches almost $100^{\circ} \mathrm{C}$ in the left-hand side. This temperature distribution can be easily explained by the fact that the liquid heats up progressively in the film as its residence time in contact with the wall increases and ends boiling. Additionally, the images of the fluorescence field are averaged over a relatively long period of time (100 images takes about $10 \mathrm{~s}$ for their acquisition) but the HS camera reveals that the bubble bursts happen only intermittently, suggesting that the temperature in the liquid film may fluctuate and have at some places an average below the boiling point. It should also be noted that the contribution of secondary droplets in this picture may be less accurately taken into account than the liquid film, since most of the secondary are ejected out of the depth of field of the microscope and with a relative low occurrence. In Fig. 16b, the signal decreases rapidly with the distance to the wall, in agreement with the fact that the liquid film thickness does not exceed $0.5 \mathrm{~mm}$. During the acquisition, significant fluctuations of the fluorescence signal from one image to another were observed. These variations were induced by the unstable movement of the liquid film. To avoid any risk of saturation in one of the images in the series, the exposure time was drastically decreased resulting in a rather low signal when averaging over 100 images. In Fig. 17c, it can be seen that the uncertainty increases strongly with the distance to the wall. This uncertainty is related to the statistical convergence of the signal acquired by the cameras. Of course, there exist other sources of error such as the reference measurement, the calibration of the temperature response of the system, the pixel correspondence of the images, and the effect of the optical aberrations, whose possible contributions have been already discussed.

\section{Conclusion}

This study reports on an imagery technique for measuring the spatial distribution of the temperature in droplet streams. This technique is an extension of the point-wise two-color laser-induced fluorescence technique that has been particularly tested to measure the temperature of droplets in the past. Measurement with this new approach requires taking some precautions, for example, the nonlinearity of the optical system response and possible optical aberrations. Because of the particularly reduced size of the measurement zone, an original procedure for the pixel-bypixel correspondence of the camera images had to be developed. Tests performed on isothermal and heated droplet streams reveal that the measurement errors are moderate in controlled conditions and the measurements are consistent with previous work in the case of droplets impinging onto a heated wall.

An improvement of the measurement system could be to adapt it for time-resolved measurements in respect to the characteristic time of the droplet/wall interactions in the presented experiments with the use of a pulse laser to excite the fluorescence.

Acknowledgments This work has been supported by the French National Agency (ANR) in the frame of the research program IDHEAS (n ANR-NT09 432160).

\section{References}

Bruchhausen M, Guillard F, Lemoine F (2005) Instantaneous measurement of two-dimensional temperature distributions by 
means of two-color planar laser induced fluorescence (PLIF). Exp Fluids 38:123-131

Castanet G, Liénart T, Lemoine F (2009) Dynamics and temperature of droplets impacting onto a heated wall. Int J Heat Mass Transf 52:670-679

Coolen MCJ, Kieft RN, Rindt CCM, Van Steenhoven AA (1999) Application of 2-D LIF temperature measurements in water using a Nd:YAG laser. Exp Fluids 27:420-426

Coppeta J, Rogers C (1998) Dual emission laser induced fluorescence for direct planar scalar behavior measurements. Exp Fluids 25:1-15

Deprédurand V, Miron P, Labergue A, Wolff M, Castanet G, Lemoine F (2008) A temperature sensitive tracer suitable for two-colour laser-induced fluorescence thermometry applied to evaporating droplets. Meas Sci Technol 19:105403

Düwel I, Ge HW, Kronemayer H, Dibble R, Gutheil E, Schulz C, Wolfrum J (2007) Experimental and numerical characterization of a turbulent spray flame. Proc Combust Inst 31:2247-2255

Glowacki J (1964) Further investigations into fluorescence quenching of dyes by neutral salts. Acta Physica Polonica 15:767-780

Hiller B, Hanson RK (1990) Properties of the iodine molecule relevant to laser-induced fluorescence experiments in gas flows. Exp Fluids 10:1-11

Kim HJ, Kihm KD, Allen JS (2003) Examination of ratiometric laser induced fluorescence thermometry for microscale spatial measurement resolution. Int J Heat Mass Transf 46:3967-3974

Labergue A, Deprédurand V, Delconte A, Castanet G, Lemoine F (2010) New insight into two-color LIF thermometry applied to temperature measurements of droplets. Exp Fluids

Lavieille P, Lemoine F, Lebouché M, Lavergne G (2001) Evaporating and combusting droplet temperature measurement using two colors laser induced fluorescence. Exp Fluids 31:45-55

Lavieille P, Delconte A, Blondel D, Lebouché M, Lemoine F (2004) Non-intrusive temperature measurements using three-color laserinduced fluorescence. Exp Fluids 36:706-716
Lemoine F, Antoine Y, Wolff M, Lebouche M (1999) Simultaneous temperature and $2 \mathrm{D}$ velocity measurements in a turbulent heated jet using combined laser-induced fluorescence and LDA. Exp Fluids 26:315-323

Lu QZ, Melton LA (2000) Measurement of transient temperature field within a falling droplet. AIAA J 38:95-101

Maqua C, Castanet G, Lemoine F, Doué N, Lavergne G (2006) Temperature measurements of binary droplets using three-color laser-induced fluorescence. Exp Fluids 40:786-797

Richards CD, Richards RF (1998) Transient temperature measurements in a convectively cooled droplet. Exp Fluids 25:392-400

Sakakibara J, Adrian RJ (1999) Whole field measurement of temperature in water using two-color laser induced fluorescence. Exp Fluids 26:7-15

Sakakibara J, Adrian RJ (2004) Measurement of temperature field of a Rayleigh-Bénard convection using two-color laser-induced fluorescence. Exp Fluids 37:331-340

Shafii M, Lum C, Koochesfahani M (2010) In situ LIF temperature measurements in aqueous ammonium chloride solution during uni-directional solidification. Exp Fluids 48:651-662

Soloff SM, Adrian RJ, Liu ZC (1997) Distortion compensation for generalized stereoscopic particle image velocimetry. Meas Sci Technol 8:1441-1454

Sutton J, Fisher B, Fleming J (2008) A laser-induced fluorescence measurement for aqueous fluid flows with improved temperature sensitivity. Exp Fluids 45:869-881

Van Beeck J, Riethmuller M (1995) Non-intrusive measurements of temperature and size of single falling raindrops. Appl Opt 34:1633-1639

Walker JD (1976) Rainbow from single drops of water and other liquids. Am J Phys 44:421-433 